\title{
Math modeling of particle movements in a friction separator
}

\author{
Valentin Potapov*, Anatoliy Afanasyev, Vladimir Potapov, Sergei Frolov, and \\ Ekaterina Franyuk
}

Ural State Mining University, 620144, Ekaterinburg, Russia

\begin{abstract}
A mathematical model of particles movement of separated material in a friction separator is proposed. It includes equations of their movement at each stage of separation (along a rough inclined plane, on a curvilinear part of a springboard, impact of a particle on the surface of a drum, free motion before leaving the separation zone), and differential equations of particle movement in circulating air flow created by a rotating drum; Each of these phases of motion is described by a system of equations obtained on the basis of basic laws of mechanics. An analysis of a particle motion along aslope plane was carried out on the basis of the law on energy changes. Depending on the ratios of the recovery factor and instantaneous friction, being random variables and also the coordinates of a point impact the particle is possible to move along different trajectories with different initial conditions. The mathematical model of the process of separation of loose multicomponent materials on a friction drum-shelf separator makes it possible to investigate comprehensively the process of separation of particles on friction and elastic properties and to optimize the operating modes of the apparatus at relatively low costs without using macaques and prototypes and investigate them experimentally. On the basis of analysis of the proposed theories of particles motion, the main ways are formulated of further improvement of methodology for designing and creating friction devices.
\end{abstract}

\section{Introduction}

In the practice of separating of useful minerals the separators separating mixtures by a combination of such properties as friction and gravity, impact and friction, aerodynamic and frictional properties, etc. have found application. In the process of frictional separation two stages can be distinguished: the first stage is movement on the surface of the stratification unit; the second stage is a free (falling) whirling. Separation is the imparting to particles of the product the required speed. The task of the second stage is proper separation - ensuring the particles to fall to different distances [1-5].

\footnotetext{
* Corresponding author: valentin.potapov@m.ursmu.ru
} 


\section{Materials and Methods}

The process of motion of each particle can be described by a mathematical model, including the equations of motion at each stage of separation and differential equations of motion of particles in a circulating air flow [6-8].

For uniformity of description of the motion of a particle at each stage we introduce a system of co-ordinates xOy common to all elements of mechanical system, the beginning of which is placed at the beginning of the inclined plane, the $\mathrm{x}$ axis is directed horizontally, and the $y$ axis is vertically downward (Fig. 1). The motion of the particle begins along a plane of length $\mathrm{L}$, inclined at an angle $\beta_{\Pi}$ to the $\mathrm{x}$ axis.

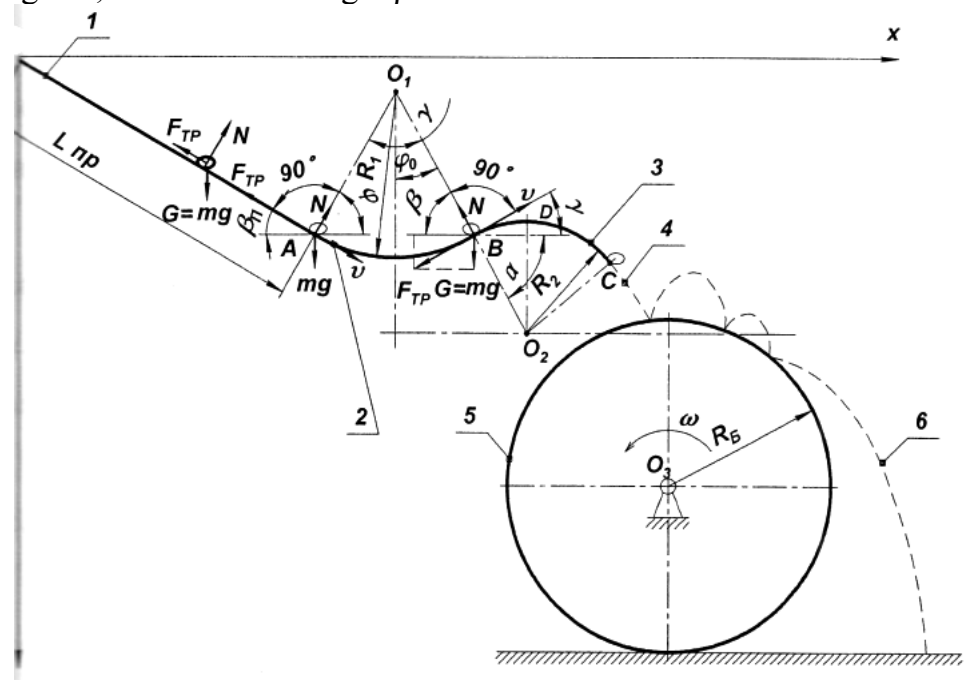

Fig. 1. Caption of the Figure 1. Below the figure.

The next section 2 (curvilinear springboard $A B$ ) will be approximately considered as an arc of a circle of radius $R_{1}$ with a central angle $\gamma_{1}$ (see Fig. 1). Section 3 of the springboard - a curved surface - is a deflector with a circle of radius $R_{2}$ with a central angle $\gamma_{2}$.

The coefficient of friction of a particle on an inclined plane and on a curvilinear springboard is assumed to be similar and denoted by $f_{\text {ck. }}$.

The free whirling of a particle generally starts from the point $C$ with a velocity $V_{\mathrm{c}}$ directed along the tangent to the arc of the circumference of the deflector 3 of the springboard at a given point. The particle is acted upon by resistance of circulating flow, which is proportional to the particle velocity in relation to the flow. The coefficient of proportionality (generally speaking, variable) will be denoted by $\square$. The coefficient depends on the aerodynamic cross section of the particle the medium viscosity and the flow velocity [3].

We define the parameters of the drum as: the coordinates of its center $C(a, b)$, the radius of the drum $R$ and its angular velocity $\omega$. If the particle touches, the drum surface during movement a shock will occur. Impact parameters: $k$ - recovery ratio and $\lambda-$ coefficient of friction at impact.

For a mathematical description of a particle motion in a drum-shelf separator it is necessary: to make equations that allow determining the particle velocity at the end of the inclined plane (at point $A$ ), at the end of the arc of the first circle (at point $B$ ) and the second circle (at point $C$ ); to write the differential equations of motion of the particle in the circulating flow and integrate them; make up the ratio to determine the velocity of the particle after its impact on the rotating drum. 
After the impact the particle again whirls in a circulating flow then again a blow may happen and whirling until the particle leaves the separation zone $(y=c)$.

The first part of the movement is an inclined shelf. When moving along this section the particle loses its initial velocity and reaches the value at its end (point $A$ ):

$$
V_{A}=\sqrt{2 g L\left(\sin \beta_{\Pi}-f_{\mathrm{c \kappa}} \cos \beta_{\Pi}\right)}
$$

where $L-$ length of the shelf, $\mathrm{m} ; \beta_{\Pi}-$ angle of its inclination, deg; $f_{\text {ск }}$-coefficient of sliding friction; $g$-acceleration of gravity, $g=9,8 \mathrm{~m} / \mathrm{s}^{2}$.

After passing through the rectilinear part the particle enters the first arc-shaped surface (curved jumping-off place) with the same speed. The motion along this surface is described in detail in [6-7], where differential dependences are given and the velocity value at the end of this section (point $B$ ) is determined:

$$
\begin{aligned}
& V_{B}^{2}=2 g L\left(\sin \beta-f_{\mathrm{cK}} \cos \beta\right) \cdot e^{2 f_{c \kappa} \gamma}+\frac{2 g R_{1}}{4 f_{C K}^{2}+1} \times
\end{aligned}
$$

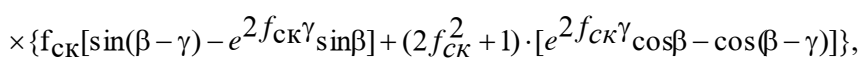

where $\gamma$ - the angle of the inclined tangent to a given curvilinear surface at the point $B ; R_{1}-$ its radius, $m$.

In the next section the particles onto a convex curvilinear surface - the deflector (Fig. 2 ). The gravitational force $G$ acts on the moving particle and the curvilinear surface response (the normal component of $N$, the tangent component is $F_{\text {тр }}$ ).

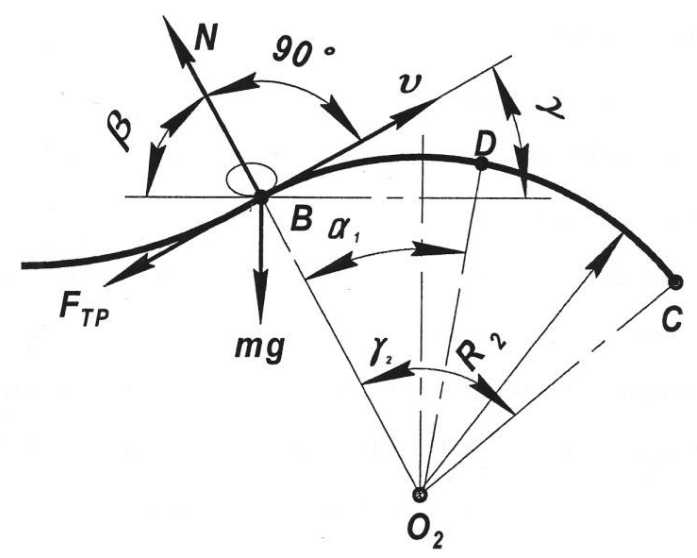

Fig. 2. Scheme of motion of particles along the surface of a curvilinear springboard.

The first corresponds to smooth continuous movement along its surface at low speeds. In the second case a detach of the particle from the surface of the deflector is possible due to the effect of the centrifugal inertia force.

The condition for detachment of a particle from jumping-off place is:

To ensure this condition it is necessary that at the upper point $D$ of the arc $B C$ the kinetic energy of the particle exceeded the work of forces resisting the motion along the $D C$ path:

$$
m g f_{\mathrm{CK}} R_{2} \int_{0}^{\alpha} \cos \alpha d \alpha<\frac{m V_{D}^{2}}{2}
$$


Using inequality (16) and the law of conservation of energy, it is quite possible to obtain conditions of the particle motion along the arc $B C$ without separation:

$$
m V_{B}^{2}>2 R_{2}\left[(1-\cos \varphi) m g+2 m g f_{\mathrm{CK}} \int_{o}^{\varphi} \cos \alpha d \alpha\right]
$$

The optimal separation mode is achieved if the arc $B C$ is bounded at point $D$. In this case the particles fall down with the initial velocity $V_{y}=0$ and the minimum velocity along the $x$ axis.

A condition without fragmentary and non-stop motion of the particle to point $D$ (found from the law of conservation of energy):

$$
m V_{B}^{2}>2 R_{2}\left[(1-\cos \varphi) m g+m g f_{\mathrm{cK}} \int_{o}^{\varphi} \cos \alpha d \alpha\right] .
$$

To ensure the motion of a particle with a separation from the deflector and without meeting it with the latter, it is necessary to fulfill the condition:

$$
\begin{gathered}
\frac{m\left(V_{B} \sin \alpha\right)^{2}}{2}>m g(1-\cos \alpha) \mathrm{R}_{2}, \\
V_{B}>\sqrt{\frac{2 g(1-\cos \varphi)}{\sin ^{2} \varphi}} R_{2} \Rightarrow \sqrt{\frac{2 g R_{2}}{(1+\cos \varphi)}} .
\end{gathered}
$$

Next the equations of free motion of a particle in a rotating air stream and its impact against the surface of the drum are solved, the initial conditions for which are the coordinates of detachment of the particle from the surface of the deflector and its velocity at this moment.

The behavior of particles in a rotating flow of the air obeying the Stokes law [3-12] is described in [8-9]. It is noted that the description of the motion in a finite form is impossible because of the complexity of systems of nonlinear differential equations:

$$
\begin{aligned}
& \ddot{x}=-\frac{\mu \cdot \dot{x}}{m}+\frac{\mu \cdot \omega \cdot R \cdot(y-b)}{m \cdot \sqrt{(x-a)^{2}+(y-b)^{2}}} \cdot \exp \left[-v \cdot \sqrt{(x-a)^{2}+(y-b)^{2}}-R\right], \\
& \ddot{y}=g-\frac{\mu \cdot \dot{y}}{m}-\frac{\mu \cdot \omega \cdot R \cdot(x-a)}{m \cdot \sqrt{(x-a)^{2}+(y-b)^{2}}} \cdot \exp \left[-v \cdot \sqrt{(x-a)^{2}+(y-b)^{2}}-R\right],
\end{aligned}
$$

where $x, y$-current coordinates of the whirling particle; $a, b$-coordinates of the point of the center of the flow circulation (the center of rotation of the drum); $\omega$ - the angular velocity of the drum; $R$ - its radius; $\mu$-coefficient of proportionality in the expression of the force resistance of the particle motion [13]:

$$
\overline{F_{c}}=-\mu \cdot \overline{V_{r}}
$$

where $\overline{V_{r}}$-the velocity of the particle relative to the movable flow. Therefore, the solution of the system of differential equations is performed by numerical methods on the PC with a step-by-step derivation of results of the integration.

The mathematical model describing the operation of this apparatus includes also the equations of impact of a particle on a rotating drum. Depending on the direction of motion 
of the particle before collision with the surface of the drum it is possible to scrape the particles in one direction or another (Fig. 3). The particle, having obtained the velocity $V_{\Pi}$ in the free flight region, after a collision acquires a new velocity $-V_{\text {от }}$.

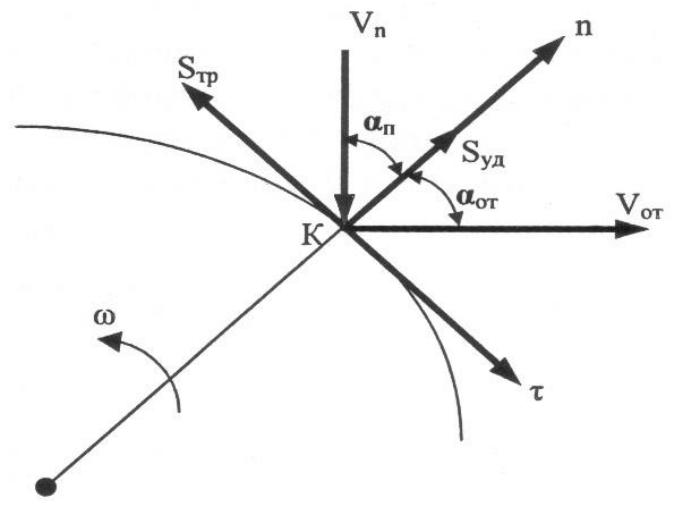

Fig. 3. Scheme of impact of a particle on a drum.

Fig. 3 denotes $S_{\text {уд }}$ the impulse of the forces acting on the particle upon impact; $S_{\text {тр }}-$ tangential composition, due to the presence of friction upon impact.

At the moment of collision with the drum at the point with the coordinates $x_{\mathrm{K}}, y_{\mathrm{K}}$ the angle $\alpha$, which determines the position of this point on the drum, can be determined from the system of equations:

$$
\left\{\begin{array}{l}
\sin \alpha=\frac{x_{\mathrm{K}}-a}{R} \\
\cos \alpha=\frac{y_{\mathrm{K}}-b}{R}
\end{array}\right.
$$

The normal vector at the point of contact is: $\vec{n}=(-\cos \alpha, \sin \alpha)$

The direction of the vector $V_{\text {от }}$ is given by the sum of the angles $\alpha$ and $\alpha_{\text {om }}$ to the vertical, therefore

$$
\begin{aligned}
& \left(V_{\text {от }}\right)_{x}=V_{\text {от }} \alpha \cos \left(\alpha+\alpha_{\text {от }}\right), \\
& \left(V_{\text {от }}\right)_{y}=V_{\text {от }} \alpha \sin \left(\alpha+\alpha_{\text {от }}\right) .
\end{aligned}
$$

These values are the initial values for a new part of the free flight of a particle in the circulating air stream.

\section{Results and Discussion}

Depending on the ratios of the recovery factor and instantaneous friction, which are random variables and also the coordinates of the impact point it is possible to move the particle along different trajectories with different initial conditions.

Thus, the described motion of particles in a friction unit is represented by finite dependences of the form (1), (2) and by differential equations. 


\section{Conclusion}

The mathematical model of the process of separation of loose multicomponent materials on a friction drum-and - cage separator makes it possible to comprehensively investigate the process of separation of particles by friction and elastic properties and to optimize the operating modes of the apparatus at relatively low costs without resorting to the manufacture of macaques and prototypes and their experimental investigation.

\section{References}

1. B.N. Kravets, Special and combined methods of enrichment, (Moscow, Nedra, 1986)

2. V.V. Olyunin, Processing of non-metallic building materials, (Moscow, Nauka, 1988)

3. F.P. Safronov, The practice of enriching asbestos ores, (Moscow, Nedra, 1975)

4. E.F Tsypin, Scientific foundations of technology of preliminary enrichment of mineral and technogenic raw materials, (2000)

5. S.A. Lyaptsev, V.Ya. Potapov, Modern problems of science and education 1, 7 (2012).

6. V.V. Potapov, Technology of development of coal deposits with its preliminary enrichment in underground conditions (2007)

7. A.I Afanasyev, V.Ya Potapov, V.V Potapov, E. E. Franyuk. Herald Zabaikal State University, 23, 24 (2017)

8. V.N Makarov, V.Ya Potapov, S.Ya Davydov, N. V Makarov. Refract and Ind Ceram, 38, 288 (2017)

9. S.A Lyaptsev, G.E Weber. Mathematical modeling of mechanical phenomena, (1993)

10. N.S. Bakhvalov, Numerical methods (Moscow, Nauka, 1973).

11. F.B Owyer, R.L Thompson, Ore sorting, 81 (1972)

12. E.F Tsypin, Yu.P. Morozov. 2nd Reg APCOM '97 Symposium on computer application and operations research in the mineral industries, 367 (1997).

13. V.V. Zubov, D.I. Simisinov, N.V. Akhlyustina, M.L. Khazin et al. Refract Ind Ceram 58, 521 (2018). 